\title{
Padrões de concordância entre múltiplos informantes na avaliação dos problemas comportamentais de adolescentes: implicações clínicas
}

\section{Agreement patterns between multiple informants in adolescents' behavioral assessment: clinical implications}

\author{
Marina Monzani da Rocha* \\ Universidade de São Paulo - USP, São Paulo, São Paulo, Brasil \\ Rafaela Almeida Ferrari** \\ Universidade de São Paulo - USP, São Paulo, São Paulo, Brasil \\ Edwiges Ferreira de Mattos Silvares*** \\ Universidade de São Paulo - USP, São Paulo, São Paulo, Brasil
}

\begin{abstract}
RESUMO
Uma avaliação abrangente inclui informações advindas de múltiplos informantes, inclusive do próprio adolescente, que oferece uma perspectiva única sobre o caso. Buscando verificar padrões de concordância entre pais e adolescentes na avaliação dos problemas dos últimos e discutir as implicações clínicas das divergências, as autoavaliações de 66 adolescentes encaminhados para atendimento psicológico, expressas na resposta ao YSR, foram comparadas às avaliações feitas pelos respectivos pais sobre os comportamentos dos filhos, expressas nas respostas ao $\mathrm{CBCL}$. O relato dos pais apontou mais problemas emocionais/comportamentais em termos de escores e na quantidade de casos que atingiram a faixa clínica dos instrumentos. Discutem-se razões para a concordância entre informantes ser maior na Escala de Externalização e implicações que discrepâncias trazem para o clínico. Conclui-se que a avaliação feita por múltiplos informantes é importante, independentemente das divergências, pois conhecer os comportamentos em diferentes contextos é fundamental para elaborar diagnósticos completos e intervenções adequadas.
\end{abstract}

Palavras-chave: Avaliação psicológica; Múltiplos informantes; Adolescente.

\begin{abstract}
A comprehensive assessment includes information obtained with multiple informants, including the adolescent himself, who offers a unique perspective of the case. In order to verify agreement patterns between parents and youths on the assessment of behavioral problems, and to discuss the clinical implications of the differences, the self-report of 66 adolescents referred for psychological treatment, on the YSR, was compared with the parents' report of their children's behavior, on the CBCL. Parents reported more behavioral problems in terms of scores and in number of clinical cases identified by the instruments. The possible reasons for the
\end{abstract}


higher agreement found between informants on the Externalizing Problems scale is discussed, as well as the clinical implications of the differences. It is concluded that the use of multiple informants on the assessment process is important, regardless of the divergences, because knowing behaviors in different contexts is crucial to elaborate complete diagnostics and adequate interventions.

Keywords: Psychological evaluation; Multiple informants; Adolescent.

\section{I ntrodução}

A avaliação dos problemas de comportamento de crianças e adolescentes é geralmente baseada em entrevistas com os pais e professores, resposta a questionários padronizados e observação dos comportamentos em contexto diagnóstico (BECKER, et al., 2004; COSTELLO; EGGER; ANGOLD, 2005). Entretanto, a maneira como os pais percebem e se pronunciam sobre seus filhos pode ser influenciada por diversas variáveis, como, por exemplo, a presença ou histórico de psicopatogias parentais (KOLKO; KAZDIN, 1993; FOLEY; et al., 2005); o tipo de problema apresentado pela criança ou adolescente, visto que alguns problemas infanto-juvenis, como as dificuldades internalizantes, são mais difíceis de serem observados e, portanto, os pais podem ter maior dificuldade em percebê-los e, por conseguinte, reportá-los (ACHENBACH; MCCONAUGHY; HOWELL, 1987; DUHIG, et al., 2000; KERR; LUNKENHEIMER; OLSO 2007), enquanto que as dificuldades externalizantes, são mais facilmente percebidas pelos adultos, sendo frequentemente apontadas como o principal motivo pela procura por atendimento na área de saúde mental (SOURANDER, et al, 2001; MARINHO, 2005; GAUY; GUIMARÃES, 2006); e o tempo de exposição aos comportamentos da criança/adolescente, já que é cada vez mais frequente as crianças passarem pouco tempo em contato com os pais e mães que, em função do trabalho e outras atividades, deixam-nas, muitas vezes, aos cuidados de terceiros (MOREIRA; BIASOLI-ALVES, 2007). Tais restrições, aliadas ao fato dos comportamentos variarem em função dos contextos nos quais são apresentados, faz com que se assuma que para fazer uma avaliação abrangente dos tipos e da extensão dos problemas apresentados, é importante obter-se informações com múltiplos informantes (DE LOS REYES; KAZDIN, 2005; FERDINAND; VAN DER ENDE; VERHULST, 2006; SALBACH-ANDRAE; LENZ; LEHMKUHL, 2009). Tal recomendação é válida principalmente se considerarmos que cada informante oferece uma perspectiva única sobre o comportamento analisado, o que certamente enriquece a visão global do caso e auxilia o profissional a tomar decisões (SMITH, 2007).

A avaliação que a própria criança ou adolescente faz sobre seus comportamentos pode trazer contribuições relevantes para a 
compreensão dos quadros apresentados e, por isso, também deve ser incluída no processo de diagnóstico (SOURANDER; HESTELÄ; HELENIUS, 1999; ZWAANSWIJK, et al., 2003; SOURANDER, 2005; ANGOLD; EGGER, 2007). Apesar de questionada no passado, estudos demonstram que existem ferramentas que permitem o uso de autoavaliação até mesmo com pré-escolares (ANGOLD; EGGER, 2007), e que os jovens que percebem seus próprios problemas apresentam maior motivação para a obtenção de tratamento, o que indica o potencial do uso de inventários auto-aplicáveis para ajudar a identificar quais adolescentes conseguirão direcionar melhor o tratamento que será oferecido (ZWAANSWIJK et al., 2003).

Discrepâncias entre os relatos dos pais e das crianças ou adolescentes sobre os comportamentos dos últimos, ainda que esperadas, são consideradas como um desafio para a compreensão do quadro clínico. Em uma meta-análise de estudos que comparavam a avaliação feita por diversos informantes, Achenbach, McConaughy e Howell (1987) encontraram baixo índice de correlação entre a autoavaliação de adolescentes e a avaliação feita pelos pais sobre eles $(r=0,25)$, principalmente se comparado ao índice de correlação encontrado no mesmo estudo para dois pais avaliando um mesmo filho $(r=0,59)$, ou dois professores avaliando uma mesma criança $(r=0,64)$. Estudos mais recentes confirmam este resultado, indicando que $O$ nível de concordância entre $O$ relato dos pais e dos adolescentes varia de baixo a moderado (SALBACH-ANDRAE; LENZ; LEHMKUHL, 2009).

Diversos pesquisadores têm buscado identificar as variáveis que podem estar relacionadas com maior ou menor concordância entre os informantes. Dentre elas é válido mencionar: sexo (VERHULST; VAN DER ENDE, 1992; BECKER, et al., 2004), idade (SMITH, 2007; SALBACH-ANDRAE; LENZ; LEHMKUHL, 2009), tipo de problema que o adolescente apresenta (COMER; KENDALL, 2004), presença de psicopatologias parentais (KOLTO; KAZDIN, 1993; BRIGGS-GOWAN; CARTER; SCHWAB-STONE, 1996; BERG-NIELSEN; VIKA; DAHL, 2003; MARTIN, et al., 2004; FOLEY, et al., 2005), nível socioeconômico (JOHNSON; WANG, 2008) e desajuste familiar (KOLTO; KAZDIN, 1993; GRILLS; OLLENDICK, 2003).

O tipo de amostra é também uma variável apontada consistentemente na literatura como tendo influência no número e na gravidade dos problemas comportamentais reportados por pais na avaliação de seus filhos, e pelos adolescentes em autoavaliação. Em trabalhos com jovens da população geral, estes apontam, em média, mais problemas de comportamento que seus pais (VERHULST; VAN DER ENDER, 1992; SAWYER; BAGHURST; MATHIAS, 1992; SOURANDER; HELSTELÄ; HELENIOS, 1999) e em trabalhos com adolescentes encaminhados para serviços de saúde mental, os pais apontam mais problemas que os adolescentes (KOLKO; KAZDIN, 
1993; HANDWERK; LARZELERE; SOPER; FRIMAN, 1999; MARTIN, et al., 2004; ROCHA; COSTA; SILVARES, 2008).

Compreender tais diferenças, principalmente no que se refere aos adolescentes encaminhados para serviços de saúde mental, é uma tarefa relevante para a análise do caso individual e para ampliar o que se sabe sobre os mecanismos das psicopatologias e das discrepâncias entre a percepção de diferentes informantes como um todo. Explicações para tais resultados geralmente relacionam o papel que os pais possuem na busca pelo atendimento como um fator significativo, sendo que eles são, na maioria do caso, os principais responsáveis (VERHUST; KOOT, 1992). Assim, a demanda pelo atendimento parte inicialmente dos pais, ainda que o adolescente considere que o problema não exista, ou que não mereça atenção clínica; ou mesmo quando o adolescente minimiza a ocorrência dos problemas indicados pelos pais como uma tentativa de evitar internações ou tratamentos mais restritivos (KOLKO; KAZDIN, 1993). Apesar de existir uma literatura substancial sobre discrepâncias entre informantes, pouco se discute sobre o impacto de tal fato na tomada de decisão clínica. Ficam sem respostas questões como, por exemplo, a) Como os profissionais de saúde mental devem interpretar os dados obtidos com os diferentes informantes? b) Qual é o problema central que vai receber o foco do tratamento? c) Qual é o melhor tratamento para cada caso? d) Quem deve ser o alvo da intervenção?

Tais questionamentos não se restringem aos clínicos, pois as divergências trazem implicações aos pesquisadores que avaliam problemas psicológicos na infância/adolescência, visto que o diagnóstico obtido pode ser diferente em função de quem foi 0 informante escolhido (KAZDIN, 1989; FOLEY et al., 2005) e, consequentemente, os resultados dos estudos podem ser influenciados por essa variável.

Considerando o exposto, o presente estudo pretende comparar o relato de adolescentes encaminhados para atendimento psicológico sobre seus próprios comportamentos com o relato dos respectivos pais sobre os comportamentos dos filhos, de maneira a verificar os padrões de concordância entre esses dois informantes, e discutir as implicações clínicas das diferenças nos relatos de múltiplos informantes.

\section{Método}

\subsection{Participantes}

Participaram do estudo 66 adolescentes, sendo 45 do sexo masculino e 21 do sexo feminino, e seus respectivos pais (58 mães e oito pais). Os jovens, com idade entre 11 e 18 anos $(M=14,13$; $D P=2,09)$, 
foram encaminhados para atendimento psicológico em duas clínicasescola da cidade de São Paulo (SP) por motivos diversos e, no momento da avaliação, ainda não tinham iniciado o tratamento psicológico. A idade dos pais variou entre 30 e 57 anos $(M=43,15$; $\mathrm{DP}=6,78)$, sendo que, no momento do nascimento do adolescente, eles tinham entre 16 e 42 anos $(M=28,62 ; D P=6,31)$. Em relação ao nível de escolaridade dos pais, $14,8 \%$ possuía o Ensino Fundamental I incompleto, 29,6\% Ensino Fundamental I incompleto ou Fundamental II incompleto, $14,8 \%$ Ensino Fundamental II completo ou Médio incompleto, 18,5\% Ensino Médio completo ou Superior incompleto e 22,2\% Ensino Superior completo.

\subsection{I nstrumentos}

"Inventário de Comportamentos para Crianças e Adolescentes entre 6 e 18 anos" - Child Behavior Checklist (CBCL/6-18 - ACHENBACH; RESCORLA, 2001) e "Inventário de Autoavaliação para Adolescentes de 11 a 18 anos" - Youth Self-Report (YSR - ACHENBACH; RESCORLA, 2001), desenvolvidos a partir do início da década de 1960 nos Estados Unidos, para avaliar comportamentos de crianças e adolescentes, sendo o primeiro inventário a partir do ponto de vista dos pais ou cuidadores e o segundo a partir do próprio adolescente. A avaliação dos itens de problemas de comportamento do $\mathrm{CBCL}$ e do YSR fornece um perfil do adolescente em oito escalas-síndromes: Ansiedade/Depressão, Retraimento/Depressão, Queixas Somáticas, Problemas de Sociabilidade, Problemas com o Pensamento, Problemas de Atenção, Violação de Regras e Comportamento Agressivo. As três primeiras são reunidas na soma de escala nomeada Escala de Internalização, as duas últimas na Escala de Externalização e todos os itens de problemas compõem a Escala Total de Problemas Emocionais/Comportamentais, sendo que, para efeito desse estudo, apenas as somas de escalas foram utilizadas em função de sua abrangência.

Os escores brutos, derivados da soma da pontuação fornecida para cada um dos itens que compõem a escala, foram transformados em escores $\mathrm{T}$ para ser possível verificar se os escores obtidos nas diferentes escalas de ambos os instrumentos atingiram a faixa clínica estabelecida para a amostra normativa americana (Escore $T \geq 60$ ) (ACHENBACH; RESCORLA, 2001).

Estudos de validação desses questionários foram desenvolvidos em diversas sociedades (YSR: RESCORLA et al., 2007a; CBCL: RESCORLA et al., 2007b). No Brasil, Bordin, Mari e Caiero (1995) realizaram um estudo de validação preliminar com a versão anterior do CBCL (ACHENBACH, 1991) a partir do qual concluíram que o instrumento é válido para avaliar a população brasileira, visto que 
apresenta alto índice de correlação com avaliação feita por um psiquiatra que não teve acesso aos dados do inventário. Atualmente, Emerich, Rocha e Silvares (2010) estão realizando uma investigação com a versão mais recente do CBCL (ACHENBACH; RESCORLA, 2001) para obter índices de validade e normas para o instrumento. No que se refere ao YSR, um projeto de validação e normatização do instrumento vem sendo desenvolvido (ROCHA, 2011), com dados preliminares que confirmam sua capacidade de discriminar os jovens encaminhados para atendimento psicológico dos jovens da população geral (ROCHA; SILVARES, 2008), além da validade convergente, estabelecida a partir da correlação dos escores obtidos com este instrumento e o "Inventário de Habilidades Sociais para Jovens" (DEL PRETTE; DEL PRETTE, 2009), e de critério, estabelecida em função da sensibilidade do instrumento em termos de produzir escores críticos em relação aos escores de referência normativa estabelecidos no estudo original norte-americano de normatização do instrumento (DEL PRETTE, et al, no prelo).

\subsection{Procedimento}

O presente estudo faz parte de um projeto maior aprovado pelo Comitê de Ética em Pesquisas com Seres Humanos do Instituto de Psicologia da Universidade de São Paulo (Prot.-1007/CEPHIP/23/04/2007) e seguiu todas as normas estabelecidas pela legislação vigente sobre ética na pesquisa com seres humanos. Os pais que buscaram atendimento psicológico para seus filhos, ou que aguardavam em fila de espera, em duas clínicas-escola de São Paulo que atendem adolescentes com queixas diversas, foram convidados a participar do estudo sobre problemas de comportamento na adolescência. Após assinar um "Termo de Consentimento Livre e Esclarecido" com informações sobre a pesquisa, o CBCL foi entregue para 0 preenchimento autônomo daqueles que apresentavam condições de leitura suficiente para tal, ou com o auxílio de um dos pesquisadores, quando necessário. Separadamente, os adolescentes também foram convidados a participar do estudo e responderam ao YSR.

Os dados obtidos com ambos os questionários foram corrigidos usando o Assessment Data Manager (Achenbach System of Empirically Based Assessment, 2006), programa de computador desenvolvido exclusivamente para essa finalidade.

\subsection{Análise Estatística}


A diferença entre os escores $T$ médios obtidos a partir das respostas dos pais ao CBCL e dos adolescentes ao YSR foi analisada através de analises de variância (ANOVA) com medidas repetidas. A correlação entre esses escores foi calculada através do coeficiente de correlação de Pearson. De acordo com o critério de Cohen para tamanho do efeito, correlações menores que 0,30 foram consideradas baixas, de 0,30 a 0,50 moderadas e maiores que 0,50, altas (COHEN, 1988). O índice de concordância Kappa foi utilizado para verificar a concordância entre os informantes quanto à classificação de um caso como clínico a partir do escore do CBCL e do YSR. Valores $p$ iguais ou inferiores a 0,05 foram considerados significativos. O programa estatístico utilizado para fazer os cálculos foi o SPSS 16.0 for Windows.

\section{Resultados}

\subsection{Correlação e comparação dos escores do CBCL e YSR}

A Tabela 1 apresenta a média e desvio padrão (DP) dos escores T obtidos pelos adolescentes nas somas de escala do CBCL e YSR, bem como o índice de correlação de Pearson ( $r$ ) entre os escores obtidos nas somas de escalas a partir da avaliação feita pelos diferentes informantes.

\begin{tabular}{l|cccc}
\hline & $\begin{array}{c}\text { Faixa Clínica } \\
\text { (CBCL e YSR) }\end{array}$ & $\begin{array}{c}\text { CBCL } \\
\text { Média (DP) }\end{array}$ & $\begin{array}{c}\text { YSR } \\
\text { Média (DP) }\end{array}$ & $r$ \\
\hline Escala de Internalização & & $66,2(7,82)$ & $59,64(9,65)$ & $0,247^{*}$ \\
Escala de Externalização & Escore T $\geq 60$ & $61,48(10,32)$ & $56,26(8,43)$ & $0,432^{* *}$ \\
Escala Total & & $64,65(9,48)$ & $58,36(8,55)$ & 0,192 \\
\hline
\end{tabular}

*p<0,05; **p<0,001.

Tabela 1: Média, Desvio Padrão (DP) e Correlação ( $r$ ) entre os Escores T obtidos a partir da avaliação pelos diferentes informantes.

O teste ANOVA com medidas repetidas foi usado para examinar a diferença entre os escores da avaliação feita pelos diferentes informantes. Os pais reportaram mais problemas que os jovens para todas as escalas analisadas, sendo a diferença entre os escores $T$ médios significativa para Escala de Internalização $\left(F_{(1,65)}=24,276\right.$; $\mathrm{p}<0,001 ; \mathrm{PO}=0,998)$, Escala de Externalização $\left(\mathrm{F}_{(1,65)}=17,618\right.$; $\mathrm{p}<0,001 ; \quad \mathrm{PO}=0,995)$ e Escala Total de Problemas Emocionais/Comportamentais $\left(F_{(1,65)}=19,779 ; p<0,001 ; P O=0,992\right)$. Pode-se, portanto, dizer, com base nas respostas dos pais ao $\mathrm{CBCL}$, que os jovens atingiram escores médios equivalentes a faixa clínica estabelecida pela amostra normativa norte-americana (Escore $\mathrm{T} \geq 60$ ) 
e, que na autoavaliação, o escore médio obtido pelos jovens ficou na faixa normal (Escore $T<60$ ) em todas as escalas.

O coeficiente de correlação de Pearson entre os escores do $\mathrm{CBCL}$ e do YSR para as somas de escala dos instrumentos foi moderado para Escala de Externalização $(r=0,432 ; p<0,001)$ e baixo para a Escala de Internalização $(r=0,247 ; p=0,046)$. Para escore total de problemas emocionais/comportamentais, a correlação entre o escores obtidos pelos jovens na avaliação dos pais e na autoavaliação foi 0,192 .

\subsection{Porcentagem de casos com escores na faixa clínica}

A porcentagem de casos que atingiu a faixa clínica na avaliação dos pais e na autoavaliação é apresentado na Figura 1.

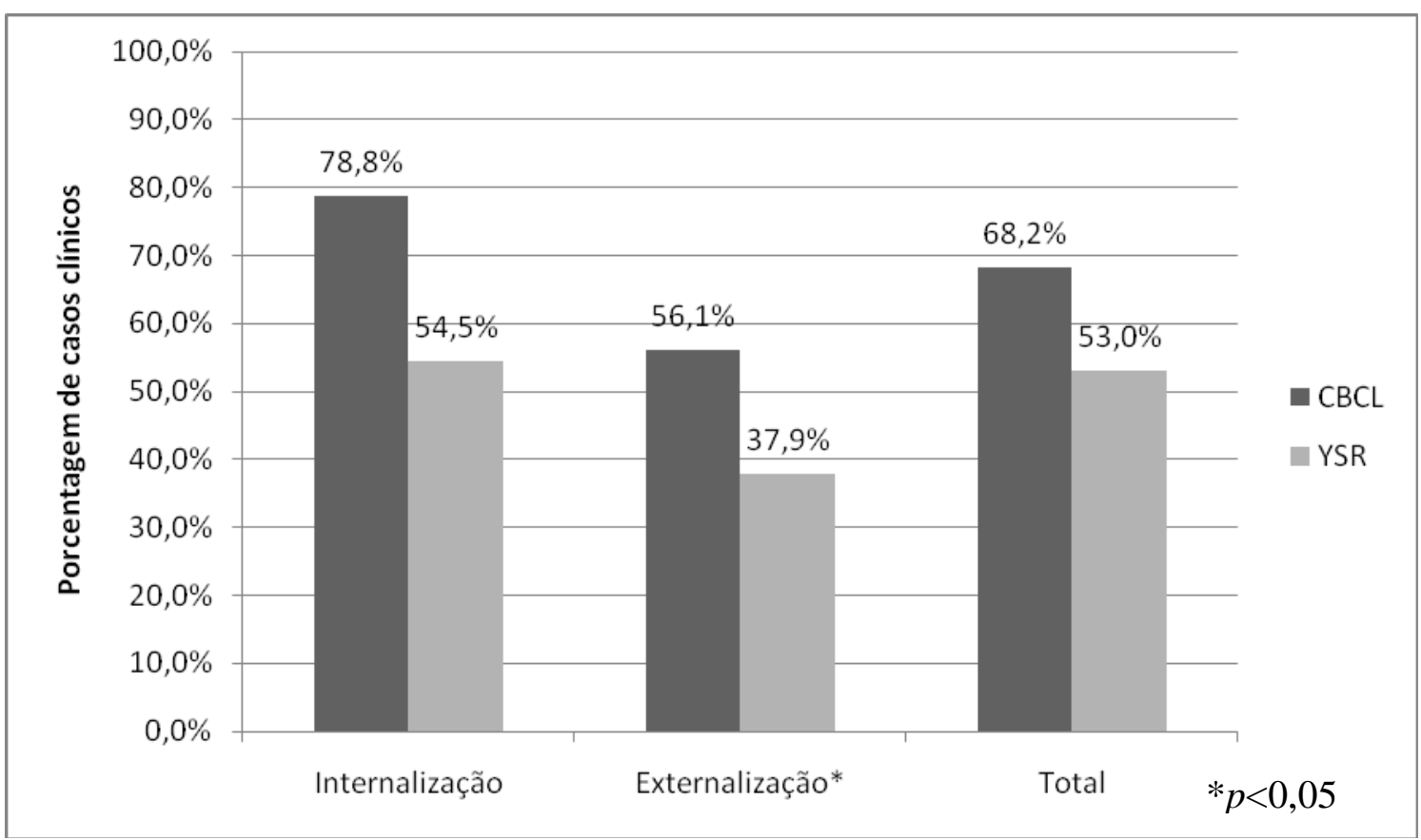

Figura 1. Porcentagem de casos com escore na faixa clínica.

Observa-se na Figura 1 que a porcentagem de casos que atingiu a faixa clínica foi maior na avaliação dos pais (CBCL) se comparada à autoavaliação (YSR) para todas as somas de escalas de problemas de comportamento. A análise Kappa foi utilizada para verificar a concordância entre os dois informantes quanto ao número de casos clínicos obtidos. Foi encontrada associação significativa apenas para a Escala de Externalização $(K=0,235 ; p=0,042)$, para a qual o índice de concordância foi satisfatório. Para a Escala de Internalização $(\mathrm{K}=0,105 ; \quad \mathrm{p}=0,322)$ e Total de Problemas 
Comportamentais/Emocionais $(K=0,132 ; \mathrm{p}=0,258)$ a concordância entre os informantes foi baixa.

A escala com maior número de casos clínicos, tanto na avaliação dos pais, quanto na dos adolescentes, foi a Escala de Internalização, na qual $78,8 \%$ dos pais e $54,5 \%$ dos adolescentes indicaram problemas em intensidade ou frequência superior ao esperado para a faixa etária. Para a Escala de Externalização, 56,1\% dos adolescentes atingiram a faixa clínica na avaliação feita pelos pais e $37,9 \%$ na autoavaliação. $\mathrm{Na}$ Escala Total, que inclui todos os itens de problemas emocionais/comportamentais analisados pelos inventários, $68,2 \%$ dos adolescentes atingiram a faixa clínica a partir da resposta do pai ao $\mathrm{CBCL}$ e $53 \%$ a partir da autoavaliação no YSR.

\section{Discussão dos resultados}

O relato feito pelos pais sobre os comportamentos de seus filhos adolescentes encaminhados para atendimento psicológico foi comparado com a autoavaliação feita pelos próprios adolescentes. Confirmou-se o que é relatado na literatura internacional e nacional em estudos com jovens encaminhados para atendimento em serviços de saúde mental (HANDWERK, et al., 1999; ACHENBACH; RESCORLA, 2001; MARTIN, et al., 2004; ROCHA; COSTA; SILVARES, 2008), visto que a avaliação feita pelos pais foi a que apontou mais problemas emocionais/comportamentais. Tal diferença entre os relatos pode ser facilmente observável a partir da comparação dos escores médios atingidos a partir da avaliação feita pelos pais, que atingiram níveis clínicos, com os escores obtidos a partir da autoavaliação dos adolescentes, dentro da faixa de normalidade do instrumento.

$O$ índice de correlação entre as respostas dadas aos dois questionários foi baixo no geral $(r=0,19$ para a Escala Total de Problemas Emocionais/Comportamentais), assim como o índice médio encontrado na meta-análise de Achenbach, McConaughy e Howell (1987) $(r=0,25)$, sendo apenas moderado para a Escala de Externalização $(r=0,43)$. Kolko e Kazdin (1993), assim como SalbachAndrae, Lenz e Luhmkuhl (2009), encontraram resultados semelhantes no que se refere à maior concordância entre os informantes para os problemas externalizantes. Parece ser consenso na literatura que, por ser mais facilmente observável, a avaliação dos problemas externalizante têm melhores índices de correlação que a dos problemas internalizantes. Ainda nesse sentido, o índice de concordância quanto a classificação de um caso como clínico foi satisfatório para a Escala de Externalização, mas baixo para Internalização e Total de Problemas Emocionais/Comportamentais, resultado novamente esperado visto que os problemas externalizantes são mais facilmente percebidas pelos adultos, sendo, 
inclusive, o principal motivo pela procura por atendimento na área de saúde (SOURANDER, et al., 2001; MARINHO, 2005; GAUY; GUIMARÃES, 2006).

Os resultados encontrados no presente estudo, consistentes com o que vêm sendo indicado na literatura nacional e internacional sobre o assunto, devem ser discutidos em termos de possíveis explicações para as diferenças encontradas entre os relatos dos pais e dos adolescentes no contexto de encaminhamento para saúde mental. As hipóteses tradicionais baseiam-se no fato de que os pais, como aqueles com o papel principal no encaminhamento para atendimento psicológico, fazem tal encaminhamento com base em suas demandas. Assim, algo que incomoda os pais naquele momento pode não ser considerado necessariamente como problemático pelo adolescente em sua autoavaliação, ou pode, até mesmo, passar despercebido por este, quando o problema não o perturba. Com um raciocínio semelhante, Kolko e Kazdin (1993) propõem que a criança ou adolescente pode minimizar, ou, até mesmo negar a existência do problema, se acredita que não há necessidade de tratamento, ou quer evitar internações ou tratamentos mais restritivos, o que parece-nos bastante plausível. Analisando por outra perspectiva, podemos pensar na hipótese dos pais exagerarem nos seus relatos, superestimando a quantidade e/ou frequência de problemas de comportamentos em seus filhos, para procurar garantir a oportunidade de que seu filho receba o tratamento desejado, visto que as vagas são limitadas nos serviços estudados, e os pais podem acreditar que casos mais graves talvez recebam algum tipo de prioridade. O mesmo foi hipotetizado por Rocha, Costa e Silvares (2008) com relação a resultados semelhantes obtidos com uma amostra de adolescentes encaminhados para atendimento específico para enurese. Por fim, não podemos esquecer que, por muitas vezes, as discrepâncias entre informantes ocorrerem em função da falta de acesso direto a alguns comportamentos (ex.: problemas internalizantes ou comportamento do adolescente na escola etc.), ou pelas alterações contextuais destes; nesse caso, os pais podem superestimar/subestimar a presença de problemas, relatando aquilo que imaginam que acontece (COMER; KENDALL, 2003). Todas essas explicações são plausíveis diante dos resultados encontrados, porém, novos estudos, com metodologias diferenciadas e incluindo outras variáveis, devem ser realizados para ampliar o conhecimento sobre os mecanismos que levam à discrepâncias entre informantes.

Implicações Clínicas: A baixa concordância entre pais e adolescentes na avaliação dos problemas dos jovens, apesar de ser esperada por refletir a opinião de informantes diferentes que a formulam, em função do comportamento do jovem em diferentes contextos (SMITH, 2007), preocupa os pesquisadores e clínicos (KAZDIN, 1989). A mais imediata se refere ao diagnóstico: Como saber se aquele adolescente 
específico necessita de atendimento psicológico se na percepção de seus pais ele apresenta problemas de comportamento, mas isso não fica evidente em sua própria avaliação? Ou o que fazer com o adolescente que reporta diversos problemas que não são identificados ou não causam preocupação em seus pais ou responsáveis? Essas questões nos remetem à importância de um diagnóstico clínico preciso e abrangente, que compreenda a vivência do adolescente nos diferentes contextos, e integre as informações provenientes dos diferentes informantes, sejam elas obtidas através de entrevistas clínicas ou de instrumentos padronizados.

No geral, os clínicos e pesquisadores interpretam cada uma das avaliações como relevantes, independentemente das discrepâncias, visto que elas expressam como aquela criança ou adolescente se comporta em cada um dos diferentes contextos (BERG-NIELSEN; VIKA; DAHL, 2003; DE LOS REYES; KAZDIN, 2005; ACHENBACH, 2006). Ferdinand, van der Ende e Verhulst (2006) sugerem que 0 profissional deve ir além de tentar determinar quem está "certo", trabalhando com todas as informações e considerando as diferenças como importantes e com significado clínico, visto que discrepâncias não são necessariamente distorções nos dados; elas podem refletir verdades subjetivas e parciais sobre a realidade. Além disso, elas podem ser parcialmente resolvidas com investigações posteriores, feitas pelo profissional por meio de entrevistas e observações comportamentais, já que não existe uma única metodologia para se fazer a avaliação psicológica (GRESHAM, et al., 2010).

Outra questão que se coloca é com relação ao planejamento da intervenção. A baixa concordância entre informantes é um fator que pode reduzir a certeza das conclusões que são tomadas na classificação do tipo de problema, estimativa da intensidade e na avaliação das mudanças. Além disso, ela pode levar a diagnósticos imprecisos (FOLEY, et al., 2005) e, até mesmo, a não adesão ao tratamento por uma das partes (WAAKTAAR, et al., 2005), que não concorda com os problemas escolhidos pelo profissional como alvo da intervenção. Considerando que estudos atuais já apontam a discrepância entre informantes como um importante fator de risco para o desenvolvimento de comportamentos problemáticos (FERDINAND; VAN DER ENDE; VERHULST, 2006) e que o jovem que reconhece seus próprios problemas tem maiores chances de aderir ao tratamento (WAAKTAAR, et al., 2005), o clínico deve buscar compreender a visão de cada um dos membros da díade paiadolescente e traçar uma estratégia de intervenção que satisfaça as necessidades do cliente. Não é função do psicólogo buscar saber se alguém está enganado ou certo; ele deve agrupar as informações obtidas através das diversas fontes e usá-las na elaboração de seu diagnóstico (FERDINAND; VAN DER ENDE; VERHULST, 2006). 
A avaliação feita a partir de múltiplos informantes é muito rica e não pode ser desvalorizada em função das discordâncias encontradas. Saber como a criança ou adolescente se comporta em cada ambiente, em cada interação, é fundamental para o profissional da saúde mental, pois, com esses dados ele tem a possibilidade de entender melhor o funcionamento daquele indivíduo e de traçar o plano de intervenção mais adequado. Além disso, esse conhecimento dos casos individuais pode ser um caminho para nos aproximarmos da compreensão do mecanismo que existe por trás das discrepâncias entre informantes no geral (WAAKTAAR, et al., 2005).

Limitações: Alguns fatores limitam as conclusões do presente estudo. A primeira delas é o número relativamente pequeno de participantes que não permite que sejam feitas análises estatísticas precisas para verificar variáveis demográficas que têm sido apontadas na literatura como possíveis interferências do resultado, como sexo e idade. Além disso, a falta de acesso ao diagnóstico clínico dos adolescentes que participaram do estudo é um déficit a ser considerado, visto que, por isso, não foi possível incluir o tipo de problema apresentado pelos adolescentes como uma das variáveis de interesse. Seria importante ter mais dados sobre a família dos adolescentes, como discórdia conjugal, nível socioeconômico, distúrbio mental ou criminalidade na família, número excessivo de filhos e superpovoamento da casa, conhecidos fatores de risco para problemas comportamentais infantis (RUTTER, 1981), que estão dentre as variáveis possivelmente associadas com discrepância entre informantes (KOLKO; KAZDIN, 1993; FOLEY, et al., 2005). Por fim, apesar de estudos preliminares indicarem a validade do CBCL (BORDIN; MARI; CAIERO, 1995) e do YSR (ROCHA; SILVARES, 2008; DEL PRETTE, et al., no prelo), ainda não há normas brasileiras para os instrumentos utilizados, o que exigiu o emprego de normas norte-americanas para comparação dos dados.

\section{Conclusão}

Os resultados confirmam os estudos anteriores que indicam grau baixo a moderado de concordância entre pais e adolescentes, o que não diminui o valor das informações obtidas através de múltiplos informantes para a elaboração do diagnóstico clínico. Apesar das percepções sobre os comportamentos serem diferentes, todas são clinicamente importantes e devem ser utilizadas pelo profissional para planejar a intervenção adequada.

\section{Referências}


ACHENBACH, T. M. Manual for the child behavior checklist and 1991 profile. Burlington, VT: University of Vermont Department of Psychiatry, 1991.

ACHENBACH, T. M.; MCCONAUGHY, S. H.; HOWELL, C. T. Child/adolescent behavioral and emotional problems: implications of cross-informant correlations for situational specificity. Psychological Bulletin, v. 101, n. 2, p. 312-332, 1987.

ACHENBACH, T. M.; RESCORLA, L. A. Manual for the ASEBA school-age forms and profiles. Burlington, VT: University of Vermont, Research Center for Children, Youths and Families, 2001. ACHENBACH, T. M. As others see us: clinical and research implications of cross-informant correlations for Psychopathology. Current Directions in Psychological Science, v. 15, n. 3, p. 9498, 2006.

ACHENBACH SYSTEM OF EMPIRICALLY BASED ASSESSMENT. Manual for the assessment data manager program (ADM). Burlington, VT: University of Vermont, Research Center for Children, Youth, \& Familie, 2006.

BECKER, A.; HAGENBERG, N.; ROESSNER, V.; WOERNER, W.; ROTHENBERGER, A. Evaluation of the self-reported SQD in a clinical setting: Do self-reports tell us more than ratings by adult informants? European Child \& Adolescent Psychiatry, v. 13, n. 2, p. II/17II24, 2004.

BERG-NIELSEN, A. V.; VIKA, A.; DAHL, A. A. When adolescents disagree with their mothers: CBCL-YSR discrepancies related to maternal depression and adolescent self-esteem. Child: Care, Health \& Development, v. 29, n. 3, p. 307-213, 2003.

BORDIN, I. A. S.; MARI, J. J.; CAEIRO, M. F. Validação da versão brasileira do "Child Behavior Checklist" (CBCL) (Inventário de Comportamentos da Infância e Adolescência): dados preliminares. Revista ABP-APAL, São Paulo, v. 17, n. 2, p. 55-66, 1995.

BRIGGS-GOWAN, M. J.; CARTER, A. S.; SCHWAB-STONE, M. Discrepancies among mother, child, and teacher reports: Examining the contributions of maternal depression and anxiety. Journal of Abnormal Child Psychology, v. 24, n. 6, p. 749-765, 1996.

COHEN, J. Statistical power analysis for the behavioral sciences, $2^{\text {nd }}$ edition. New York: Academic Press, 1988.

COMER, J. S.; KENDALL, P. C. A symptom-level examination of parent-child agreement in the diagnosis of anxious youths. Journal of the American Academy of Child and Adolescent Psychiatry, v. 43, n. 7, p. 878-886, 2004.

DE LOS REYES, A.; KAZDIN, A. E. Informant discrepancies in the assessment of childhood psychopathology: A critical review, theoretical framework, and recommendations for further study. Psychological Bulletin, v. 131, n. 4, p. 483-509, 2005. 
DEL PRETTE, Z. A. P.; DEL PRETTE, A. Inventário de habilidades sociais para adolescentes (IHSA-Del-Prette): Manual para aplicação, apuração e interpretação. São Paulo: Casa do Psicólogo, 2009.

DUHIG, A. M.; RENK, K.; EPSTEIN, M. K.; PHARES, V. Interparental agreement on internalizing, externalizing, and total behavior problems: a meta-analysis. Clinical Psychology Science and Practice, v. 7, n. 4, p. 435-453, 2000.

EMERICH, D. R.; ROCHA, M. M.; SILVARES, E. F. M. Estudo de validação preliminar do "I nventário dos Comportamentos de Crianças e Adolescentes de 6 a 18 anos" (CBCL/6-18), versão brasileira do Child Behavior Checklist. Projeto de Pesquisa submetido ao Comitê de Ética em Pesquisas com Seres Humanos do Instituto de Psicologia da Universidade de São Paulo. 2010.

FERDINAND, R. F.; VAN DER ENDE, J.; VERHULST, F. C. Prognostic value of parent-adolescent disagreement in referred sample. European Child and Adolescent Psychiatry, v. 15, n. 3, p. 156162, 2006.

FOLEY, D. L.; RUTTER, M.; ANGOLD, A.; PICKLES, A. MAES, H. M.; SILBERG, J. L.; EAVES, L. J. Making sense of informant disagreement for overanxious disorder. Journal of Anxiety Disorders, v. 19, p. 193-210, 2005.

GAUY. F. V.; GUIMARÃES, S. S. Triagem em saúde mental infantil. Psicologia: Teoria e Pesquisa, v. 22, p. 05-16, 2006.

GRESHAM, F. M.; ELLIOTT, S. N.; COOK, C. R.; VANCE, M. J.; KETTLER, R. Cross-informant agreement for ratings for social skill and problem behavior ratings: an investigation of the social skills improvement system - rating scales. Psychological Assessment, v. 22, n. 1, p. 157-166, 2010.

GRILLS, A. E.; OLLENDICK, T. H. Multiple informant agreement and the anxiety disorders interview schedule for parents and children. Journal of the American Academy of Child and Adolescent Psychiatry, v. 42, n. 1, p. 30-40, 2003.

HANDWERK, M. L.; LARZELERE, R. E.; SOPER, S. H.; FRIMAN, P. C. Parent and child discrepancies in reporting severity of problems behaviors in three out-of-home settings. Psychological Assessment, v. 11, n. 1, p. 14-23, 1999.

JOHNSON, S. B.; WANG, C. Why do adolescents say they are less healthy than their parents think they are? The importance of mental health varies by social class in a nationally representative sample. Pediatrics, v. 121, n. 2, p. 307-313, 2008.

KAZDIN, A. Identifying depression in children: a comparison of alternative selection criteria. J ournal of the American Academy of Child and Adolescent psychiatry, v. 17, p. 437-454, 1989.

KERR, D. C. R.; LUNKENHEIMER, E. S.; OLSON, S. L. Assessment of child problem behaviors by multiple informants: a longitudinal study 
from preschool to school entry. The Journal of Child Psychology and Psychiatry, v. 48, n. 10, p. 967-975, 2007.

KOLKO, D. J.; KAZDIN, A. E. Emotional/Behavioral problems in clinic and nonclinic children: Correspondence among child, parent and teacher reports. Journal of Child Psychology and Psychiatry, v. 34, n. 6, p. 991-1006, 1993.

MARTIN, J. K.; FORD, C. B.; DYER-FRIEDMAN, J.; TANG, J.; HUFFMAN, L. C. Patterns of agreement between parent and child ratings of emotional and behavioral problems in a outpatient clinical setting: When children endorse more problem. Developmental and Behavioral Pediatrics, v. 25, n. 3, p. 150-155, 2004.

MARINHO, M. L. Um programa estruturado para o treinamento de pais. In: CABALLO, V. E.; SIMÓN, M. A. (Org.). Manual de psicologia clínica infantil e do adolescente: transtornos específicos. São Paulo: Livraria Santos, 2005. p. 417-443.

ROCHA, M. M. Autoavaliação de competências e problemas de comportamento entre adolescentes brasileiros: Um estudo de validação do Inventário de Autoavaliação para Jovens (YSR/2001). 2011. Exame de Qualificação (Doutorado em Psicologia Clínica) Programa de Pós-Graduação em Psicologia Clínica, Instituto de Psicologia, Universidade de São Paulo, 2011.

ROCHA, M. M.; COSTA, N. J.; SILVARES, E. F. M. Changes in parents' and self-reports of behavioral problems in Brazilian adolescents after behavioral treatment with urine alarm for nocturnal enuresis. I nternational Brazilian J ournal of Urology, v. 34, n. 6, p. 749757, 2008.

ROCHA, M. M.; SILVARES, E. F. M. 2008. Perfil comportamental de adolescentes indicados e não indicados para atendimento psicológico: capacidade discriminativa do YSR. In: XVII ENCONTRO ANUAL DA ASSOCIAÇÃO BRASILEIRA DE PSICOTERAPIA E MEDICINA COMPORTAMENTAL, 17, 2008, Campinas. Resumos dos trabalhos apresentados no Encontro de 2008 em Campinas. São Paulo: Associação Brasileira de Psicoterapia e Medicina Comportamental, 2008. p. 248-250.

RESCORLA, L. A.; ACHENBACH, T. M.; IVANOVA, M. Y.; DUMENCI, L.; ALMQVIST, F.; BILENBERG, N. Epidemiological comparison of problems and positive qualities reported by adolescents in 24 countries. Journal of Consulting and Clinical Psychology, v. 75, n. 2, p. 351-358, 2007a.

RESCORLA, L. A.; ACHENBACH, T. M.; IVANOVA, M; DUMENCI, L; ALMQVIST, $\mathrm{F} ;$ BILENBERG, $\mathrm{H}$. Behavioral and emotional problems reported by parents of children ages 6 to 16 in 31 societies. Journal of Emotional and Behavioral Disorders, v. 15, n. 3, p. 130-142, 2007b. 
SALBACH-ANDRAE, H.; LENZ, K.; LEHMKUHL, U. Patterns of agreement among parent, teacher and youth ratings in referred sample. European Psychiatry, v. 24, p. 345-351, 2009.

SAWYER, M. G.; BAGHURST, P.; MATHIAS, J. Differences between informants's reports describing emotional behavioural problems in community and clinic-referred children: a research note. Journal of Child Psychology and Psychiatry, v. 33, n. 2, p. 441-449, 1992.

SMITH, S. R. Making sense of multiple informants in child and adolescent psychopathology: a guide for clinicians. Journal of Psychoeducational Assessment, v. 25, n. 2, p. 139-149, 2007.

SOURANDER, A.; HELSTELÄ, L.; HELENIUS, H. Parent-adolescent agreement on emotional and behavioral problems. Social Psychiatry and Psychiatric Epidemiology, v. 34, p. 657-663, 1999.

SOURANDER, A.; HELSTELÄ, L.; RISTKARI, T.; IKÄHEIMO, K.; HELENIUS, H.; PIHA, J. Child and adolescent mental health service use in Finland. Social Psychiatry and Psychiatric Epidemiology, v. 36, n. 6, p. 294-298, 2001.

SOURANDER, A.; HELSTELÄ, L. Childhood predictors of externalizing and internalizing problems in adolescence: a prospective follow-up study from age 8 to 16 . European Child and Adolescent Psychiatry, v. 14, n. 8, p. 415-423, 2005.

VERHULST, F. C.; VAN DER ENDE, J. Agreement between parents' reports and adolescents' self-reports of problem behavior. J ournal of Child Psychology and Psychiatry, v. 33, n. 6, p. 1011-1023, 1992. WAAKTAAR, T.; BORGE, A. I. H.; CHRISTIE, H. J.; TORGERSEN, S. Youth-parent consistencies on ratings of difficulties and prosocial behavior: Exploration of an at-risk sample. Scandinavian J ournal of Psychology, v. 46, n. 2, p. 179-188, 2005.

ZWAANSWIJK, M.; VAN DER ENDE, J.; VERHAAK, P. F.; BENSING, J. M.; VERHULST, F. C. Factors associated with adolescent mental health service need and utilization. Journal of the American Academy for Child and Adolescent Psychiatry, v. 42, n. 6, p. 692-700, 2003.

Endereços para correspondência

Marina Monzani da Rocha

Av. Prof. Mello Moraes, 1721, CEP 05508-030, São Paulo-SP, Brasil

Endereço eletrônico: marinamonzani@gmail.com

Rafaela Almeida Ferrari

Av. Prof. Mello Moraes, 1721, CEP 05508-030, São Paulo-SP, Brasil

Endereço eletrônico: ferrarirafaela@yahoo.com.br

Edwiges Ferreira de Mattos Silvares

Av. Prof. Mello Moraes, 1721, CEP 05508-030, São Paulo-SP, Brasil

Endereço eletrônico: efdmsilv@usp.br 
Marina Monzani da Rocha, Rafaela Almeida Ferrari, Edwiges Ferreira de Mattos Silvares Padrões de conc. entre múltiplos inform. na aval. dos probl. comp. de adolescentes

Recebido em: 30/06/2010

Reformulado em: 09/11/2010

Aceito para publicação em: 11/11/2010

Acompanhamento do processo editorial: Deise Mancebo

\section{Notas}

*Doutoranda do Programa de Pós Graduação em Psicologia Clínica da Universidade de São Paulo, SP, Brasil.

**Mestranda do Programa de Pós Graduação em Psicologia Clínica da Universidade de São Paulo, SP, Brasil.

***Profa. Titular do Departamento de Psicologia Clínica da Universidade de São Paulo, SP, Brasil. 